\title{
STRUCTURE OF COMPLEX LINEAR DIFFERENTIAL EQUATIONS ${ }^{1}$
}

\author{
BY
}

\author{
MARGALIT RONEN
}

\begin{abstract}
In this paper homogeneous linear differential equations in the complex domain are considered. Relations between (a) properties of the zeros of solutions, (b) factorization of the equation into linear factors, and (c) nonvanishing of corresponding Wronskians are proved.
\end{abstract}

1. Introduction. This paper deals with a homogeneous linear equation of order $\boldsymbol{n}$ :

$$
L y(z)=y^{(n)}(z)+p_{n-1}(z) y^{(n-1)}(z)+\cdots+p_{0}(z) y(z)=0,
$$

where $p_{i}(z)$ are analytic functions regular in the simply connected domain $D$, $\infty \notin D, i=0,1, \ldots, n-1$.

Equation (1) is called disconjugate in $D$ if no (nontrivial) solution of (1) has $n$ zeros in $D$. (The zeros are counted by their multiplicity.)

Disconjugate equations were discussed in several papers; we shall refer to the works of Nehari [5], Lavie [3], [4] and Kim [2].

Real equations of the form (1) which are disconjugate were discussed in many papers; we shall mainly use the work of Pólya [7] and the summary of Coppel [1].

Equation (1) will be called disconjugate at two points in $D$ if there is no (nontrivial) solution such that the sum of the multiplicities of its zeros at two points is greater than or equal to $n$.

On the real axis the properties of disconjugacy and disconjugacy at two points are equivalent. For example, equation (1) with continuous coefficients is disconjugate on the half-open interval $[a, b)$ if and only if it is disconjugate at two points in $[a, b)$. (See $[1, \mathrm{pp} .87-100]$.)

In the complex case the two notions are not equivalent. For any given simply connected domain $D, \infty \notin D$, the class of equations which are disconjugate at two points in $D$ is larger than the class of equations which are disconjugate in $D$. We show this by the following example.

EXAMPLE 1. Consider the equation $y^{\prime \prime \prime}(z)-4 y^{\prime \prime}(z)+3 y^{\prime}(z)=0$, whose solutions are $1, e^{z}, e^{3 z}$. This equation has a solution with a double zero at $z_{1}$ and a simple zero at $z_{2}$ if:

$$
\left|\begin{array}{ccc}
1 & e^{z_{1}} & e^{3 z_{1}} \\
0 & e^{z_{1}} & 3 e^{3 z_{1}} \\
1 & e^{z_{2}} & e^{3 z_{2}}
\end{array}\right|=e^{z_{1}+3 z_{2}}-3 e^{3 z_{1}+z_{2}}+2 e^{4 z_{1}}=0
$$

Received by the editors November 4, 1980.

1980 Mathematics Subject Classification. Primary 34C10; Secondary 30 C55.

Key words and phrases. Disconjugacy, disconjugacy at two points, univalent functions.

${ }^{1}$ This work is part of a D.Sc. Dissertation done under the supervision of Professor B. Schwarz at the Technion-I.I.T. I wish to thank him and Dr. U. Elias for their help in the preparation of this paper. 
Multiplying by $e^{-4 z_{1}}$ we get

$$
x^{3}-3 x+2=(x-1)^{2}(x+2)=0 ; \quad x=e^{z_{2}-z_{1}} .
$$

Thus, the equation is disconjugate at two points in any domain for which

$$
e^{z_{2}-z_{1}} \neq 1=e^{2 \pi k i} \text { and } e^{z_{2}-z_{1}} \neq-2=e^{\ln 2+(1+2 k) \pi i}
$$

for example, in the rectangle $|\operatorname{Re} z|<\frac{1}{2} \ln 2,|\operatorname{Im} z|<\pi$. On the other hand the solution $y(z)=1-e^{3 z}$ has zeros at $0,2 \pi i / 3,-2 \pi i / 3$. Therefore in this rectangle the equation is disconjugate at two points but is not disconjugate.

Since the disconjugacy properties remain invariant under conformal mapping, such an example exists in any simply connected domain.

We denote by $y_{1}(z, s), y_{2}(z, s), \ldots, y_{n}(z, s)$ the basis of solutions of (1) which is determined by the initial conditions

$$
y_{i}^{(j)}(s)=\delta_{i, n-j} ; \quad s \in D ; i=1,2, \ldots, n ; j=0,1, \ldots, n-1 .
$$

Note that $y_{k}(z, s), k=1,2, \ldots, n$, are analytic functions both with respect to the variable $z$ and parameter $s$. From now on when we say that $f(z, s)$ is analytic in $D$, we mean that for every fixed $s$ in $D, f(z, s)$ is an analytic function of $z$.

The following theorem is proved in this paper:

THEOREM 1. The following four properties of equation (1) are equivalent:

1. The equation is disconjugate at two points in $D$.

2. For every $z, s \in D, z \neq s$;

$$
W_{k}(z, s)=W\left(y_{1}(z, s), y_{2}(z, s), \ldots, y_{k}(z, s)\right) \neq 0,
$$

$$
k=1,2, \ldots, n
$$

where $W$ denotes the Wronskian.

3. For every $s \in D$, the equation can be factorized:

$$
L y(z)=\left(\frac{d}{d z}+r_{n}(z)\right) \ldots\left(\frac{d}{d z}+r_{k}(z)\right) \ldots\left(\frac{d}{d z}+r_{1}(z)\right) y(z) .
$$

Here the functions $r_{k}(z)$ are regular in $D$, except at $z=s$ where each of them has a simple pole

$$
r_{k}(z)=-(n-2 k+1) /(z-s)+\ldots
$$

4. For every $s \in D$ the equation can be factorized:

$$
\begin{aligned}
L y(z)= & \frac{f_{1}(z, s) f_{2}(z, s) \ldots f_{n}(z, s)}{(z-s)^{n-1}} \\
& \cdot \frac{d}{d z}\left[\frac{(z-s)^{2}}{f_{n}(z, s)} \frac{d}{d z}\left[\ldots \frac{d}{d z}\left(\frac{(z-s)^{2}}{f_{2}(z, s)} \frac{d}{d z} \frac{y(z)}{f_{1}(z, s)(z-s)^{n-1}}\right) \ldots\right]\right)
\end{aligned}
$$

Here the functions $f_{k}(z, s)$ are analytic and different from zero in $D$.

This theorem in the complex domain is analogous to a well-known result of Pólya [7]. Pólya's result states that a real homogeneous linear equation of order $n$ is disconjugate in the interval $I$ if and only if it has the factorization

$$
L y(z)=\rho_{n}(x)\left(\rho_{n-1}(x) \ldots\left(\rho_{1}(x)\left(\rho_{0}(x) y(x)\right)^{\prime}\right)^{\prime} \ldots\right)^{\prime}=0,
$$


where

$$
\rho_{i}(x) \in C^{n-i}, \quad \rho_{i}(x)>0, \quad i=0,1, \ldots, n .
$$

2. Proof of Theorem 1. We start with some definitions and lemmas that will be required in the proof.

We say that a solution $y(z)$ has the property $(\alpha, i, \beta, j), \alpha \neq \beta, \alpha, \beta \in D$, if it has a zero of multiplicity not smaller than $i$ at $\alpha$, and a zero of multiplicity not smaller than $j$ at $\beta$. In the case where the location of the zeros is insignificant we say that $y(z)$ has the property $(i, j)$.

LEMMA 1. Equation (1) has a solution with the property $(\alpha, k, \beta, n-k)$ if and only if $W_{n-k}(\beta, \alpha)=0$.

Proof. A solution of (1) has a zero of order $k$ at $\alpha$ if and only if it is a linear combination of $y_{1}(z, \alpha), y_{2}(z, \alpha), \ldots, y_{n-k}(z, \alpha)$. Such a linear combination has a zero of order $n-k$ at $\beta$ if and only if the determinant of the system is zero, i.e. $W_{n-k}(\beta, \alpha)=0$.

COROllary. $W_{n-k}(\beta, \alpha)=0$ if and only if $W_{k}(\alpha, \beta)=0$.

This follows from Lemma 1 and the fact that the properties $(\alpha, k, \beta, n-k)$ and $(\beta, n-k, \alpha, k)$ are the same. In fact one can prove

$$
W_{k}(\beta, \alpha)=W_{n-k}(\alpha, \beta) / W_{n}(\alpha, \beta) \text {. }
$$

Similar relations are proved in [6].

Let $y_{1}(z, s), y_{2}(z, s), \ldots, y_{n}(z, s)$ be the basis of solutions of (1) which is determined by the initial conditions (2). We define the following operators:

$$
\begin{aligned}
& L_{k} y(z)=W\left(y_{1}(z, s), y_{2}(z, s), \ldots, y_{k}(z, s), y(z)\right) / W_{k}(z, s) \\
& \quad k=1,2, \ldots, n . \\
& \begin{array}{l}
Q_{k} u(z)=\frac{W_{k}(z, s)}{W_{k-1}(z, s)} \frac{d}{d z}\left(\frac{W_{k-1}(z, s)}{W_{k}(z, s)} u(z)\right) ; \\
k=1,2, \ldots, n ; \quad W_{0} \equiv 1 .
\end{array}
\end{aligned}
$$

Note that $L_{1} y(z)=Q_{1} y(z)$, because they are both equal to $y^{\prime}(z)-\left(y_{1}^{\prime} / y_{1}\right) y(z)$.

LEMMA 2. If for $z \neq s, W_{k}(z, s)$ and $W_{k-1}(z, s)$ are different from zero in $D$, then

$$
L_{k} y(z)=Q_{k} L_{k-1} y(z) \text {. }
$$

Proof. Both sides of (9) are linear differential equations of order $k$ with leading coefficient 1 and have the same solutions $y_{1}(z, s), y_{2}(z, s), \ldots, y_{k}(z, s)$; hence they are equal. (See [1, p. 87]).

LEMMA 3. Let $Q_{k}$ be the operator defined in (8). If for $z \neq s, W_{k}(z, s) \neq 0$ and $W_{k-1}(z, s) \neq 0$, in $D$, then

$$
Q_{k}=d / d z+r_{k}(z),
$$


where the function $r_{k}(z)$ is regular in $D$, except at $z=s$, and has a simple pole at $z=s$ :

$$
r_{k}(z)=-(n-2 k+1) /(z-s)+\ldots
$$

Proof. The solution $y_{i}(z, s)$ satisfies (2), and therefore

$$
y_{i}(z, s)=(z-s)^{n-i} /(n-i) !+C_{i, n}(z-s)^{n}+\ldots
$$

Hence, the first term in the Taylor series of $W_{k}(z, s)$ is

$$
\begin{aligned}
W\left(\frac{(z-s)^{n-1}}{(n-1) !}, \frac{(z-s)^{n-2}}{(n-2) !}, \ldots, \frac{(z-s)^{n-k}}{(n-k) !}\right) \\
\quad=\frac{W\left((z-s)^{n-1}, \ldots,(z-s)^{n-k}\right)}{(n-1) !(n-2) ! \cdots(n-k) !}
\end{aligned}
$$

It is easily verified that

$$
W\left(r y_{1}, r y_{2}, \ldots, r y_{k}\right)=r^{k} W\left(y_{1}, y_{2}, \ldots, y_{k}\right),
$$

where $r(z)$ is analytic in $D$. Hence

$$
\begin{aligned}
& W\left((z-s)^{n-1},(z-s)^{n-2}, \ldots,(z-s)^{n-k}\right) \\
& \quad=(z-s)^{k(n-k)} W\left((z-s)^{k-1},(z-s)^{k-2}, \ldots, 1\right) \\
& \quad=(-1)^{k(k-1) / 2}(k-1) !(k-2) ! \cdots 2 !(z-s)^{k(n-k)} .
\end{aligned}
$$

We obtain

$$
W_{k}(z, s)=A_{k}(z-s)^{k(n-k)}+\ldots ; \quad A_{k} \neq 0 .
$$

Thus, it follows from (8) and (11) that

$$
r_{k}(z)=\left(\frac{W_{k-1}}{W_{k}}\right)^{\prime} /\left(\frac{W_{k-1}}{W_{k}}\right)=-\frac{(n-2 k+1)}{(z-s)}+\ldots
$$

LEMMA 4. If

$$
v_{k}(z)=\exp \int^{z}\left(r_{k-1}(\zeta)-r_{k}(\zeta)\right) d \zeta ; \quad k=1,2, \ldots, n ; r_{0} \equiv 0,
$$

or, equivalently, if

$$
-r_{k}(z)=\frac{v_{1}^{\prime}(z)}{v_{1}(z)}+\frac{v_{2}^{\prime}(z)}{v_{2}(z)}+\cdots+\frac{v_{k}^{\prime}(z)}{v_{k}(z)} ; \quad k=1,2, \ldots, n,
$$

then

$$
v_{1} v_{2} \ldots v_{n} \frac{d}{d z} \frac{1}{v_{n}} \frac{d}{d z} \ldots \frac{1}{v_{2}} \frac{d}{d z} \frac{y(z)}{v_{1}}=\left(\frac{d}{d z}+r_{n}\right) \ldots\left(\frac{d}{d z}+r_{1}\right) y(z) .
$$

PRoof. The proof is straightforward computation (see [1, pp. 92-93]). 
LEMMA 5. If (1) has a representation

$$
\begin{aligned}
L y(z)= & v_{1} v_{2} \ldots v_{n} \frac{d}{d z} \frac{1}{v_{n}} \frac{d}{d z} \ldots \frac{1}{v_{2}} \frac{d}{d z} \frac{y(z)}{v_{1}} \\
\stackrel{\text { def }}{=} & \frac{f_{1}(z, s) f_{2}(z, s) \ldots f_{n}(z, s)}{(z-s)^{n-1}} \\
& \cdot \frac{d}{d z}\left(\frac{(z-s)^{2}}{f_{n}(z, s)} \frac{d}{d z}\left[\ldots \frac{d}{d z}\left(\frac{(z-s)^{2}}{f_{2}(z, s)} \cdot \frac{d}{d z} \frac{y(z)}{f_{1}(z, s)(z-s)^{n-1}}\right) \ldots\right]\right),
\end{aligned}
$$

where the functions $f_{k}(z, s)$ are analytic and different from zero in $D$, then there exists a basis of solutions of $(1), w_{1}(z, s), w_{2}(z, s), \ldots, w_{n}(z, s)$, such that: $w_{i}(z, s)$ has a zero of exact order $n-i$ at $s, i=1,2, \ldots, n$, and

$$
W\left(w_{1}(z, s), w_{2}(z, s), \ldots, w_{k}(z, s)\right) \neq 0 \text { in } D,
$$

for $z \neq s$, and $k=1,2, \ldots, n$.

Proof. Without loss of generality we may assume that $f_{k}(s, s)=1, k=$ $1,2, \ldots, n$. The solutions of (5) are given explicitly by (see [1, p. 97]):

$$
\begin{aligned}
& w_{1}(z, s)=(z-s)^{n-1} f_{1}(z, s)=v_{1} \\
& w_{2}(z, s)=(z-s)^{n-1} f_{1}(z, s) \int^{z} \frac{f_{2}(\zeta, s)}{(\zeta-s)^{2}} d \zeta=v_{1} \int^{z} v_{2} d \zeta, \\
& \vdots \\
& w_{k}(z, s)=(z-s)^{n-1} f_{1}(z, s) \int^{z} \frac{f_{2}\left(\zeta_{1}, s\right)}{\left(\zeta_{1}-s\right)^{2}} \ldots \\
&=v_{1} \int^{z} v_{2} \ldots \int^{\zeta_{k-2}} v_{k} d \zeta_{k-1} \ldots d \zeta_{1} ; \quad k=3, \ldots, n, \\
&
\end{aligned}
$$

and

$$
\begin{aligned}
W\left(w_{1}(z, s)\right. & \left., w_{2}(z, s), \ldots, w_{k}(z, s)\right)=v_{1}^{k} v_{2}^{k-1} \ldots v_{k} \\
= & \left((z-s)^{n-1} f_{1}(z, s)\right)^{k}\left(\frac{f_{2}(z, s)}{(z-s)^{2}}\right)^{k-1} \ldots\left(\frac{f_{k}(z, s)}{(z-s)^{2}}\right) \\
= & (z-s)^{k(n-k)} f_{1}^{k}(z, s) f_{2}^{k-1}(z, s) \ldots f_{k}(z, s), \quad k=1,2, \ldots, n .
\end{aligned}
$$

$w_{i}(z, s)$ are regular functions because they are solutions of the linear differential equation (1), which has regular analytic coefficients in a simply connected domain D. 
Note that

$$
\begin{gathered}
\int^{z} \frac{1}{\left(\zeta_{1}-s\right)^{2}} \cdots \int^{\zeta_{k-2}} \frac{1}{\left(\zeta_{k-1}-s\right)^{2}} \int^{\zeta_{k-1}} \frac{1}{\left(\zeta_{k}-s\right)^{2}} d \zeta_{k} d \zeta_{k-1} \ldots d \zeta_{1} \\
=\frac{(-1)^{k}}{k !(z-s)^{k}}+\sum_{\nu=0}^{k-1} \frac{C_{\nu}}{(z-s)^{\nu}}
\end{gathered}
$$

where $C_{\nu}$ are constants. Now it follows from (12)-(14) that $w_{i}(z, s)$ has a zero of exact order $n-i$ at $z=s$, and for $z \neq s$ the Wronskians are different from zero in D.

LEMMA 6. Let $u_{1}(z, s), u_{2}(z, s), \ldots, u_{n}(z, s)$ be solutions of $(1)$ such that $u_{i}(z, s)$ has a zero of exact order $n-i$ at $z=s, i=1,2, \ldots, n$. If $W\left(u_{1}(z, s), \ldots, u_{k}(z, s)\right) \neq 0$, for $z \in D, z \neq s, 1 \leqslant k \leqslant n$, then $W_{k}(z, s) \neq 0$ for $z \in D, z \neq s$.

ProOF.

$$
\begin{aligned}
& y_{1}(z, s)=u_{1}(z, s) / u_{1}^{(n)}(s, s), \\
& y_{2}(z, s)=\left(u_{2}(z, s)-u_{2}^{(n-1)}(s, s) y_{1}(z, s)\right) / u_{2}^{(n-2)}(s, s), \\
& y_{3}(z, s)=\frac{\left(u_{3}(z, s)-u_{3}^{(n-1)}(s, s) y_{1}(z, s)-u_{3}^{(n-2)}(s, s) y_{2}(z, s)\right)}{u_{3}^{(n-3)}(s, s)},
\end{aligned}
$$

and so on. It is obvious that

$$
W_{k}(z, s)=\frac{W\left(u_{1}(z, s), u_{2}(z, s), \ldots, u_{k}(z, s)\right)}{u_{1}^{(n-1)}(s, s) u_{2}^{(n-2)}(s, s) \ldots u_{k}^{(n-k)}(s, s)} .
$$

That completes the proof of this lemma.

Using Lemmas 1-6 we now prove Theorem 1.

Proof. (i) The equivalence of properties 1 and 2 of Theorem 1 follows immediately from Lemma 1.

(ii) The equivalence of properties 3 and 4 follows from Lemma 4: $r_{k}(z)$ are now as in property $3, k=1,2, \ldots, n$, and $v_{1}(z, s)=f_{1}(z, s)(z-s)^{n-1}, v_{k}(z, s)=$ $f_{k}(z, s) /(z-s)^{2}, k=2, \ldots, n$.

(iii) Property 2 implies property 3: If for $z \neq s, W_{k}(z, s) \neq 0, k=1,2, \ldots, n$, then by Lemma 2, equation (1) has the factorization

$$
L y(z)=Q_{n} Q_{n-1} \ldots Q_{1} y(z)
$$

where $Q_{k}, k=1,2, \ldots, n$, are defined in (8). Hence using Lemma 3 we get that equation (1) has the factorization (4).

(iv) Property 4 implies property 2: If equation (1) has the factorization (5), then by Lemma 5 the equation has a basis of solutions $w_{1}(z, s), w_{2}(z, s), \ldots, w_{n}(z, s)$, where $w_{k}(z, s)$ has a zero of exact order $n-k$, at $z=s, k=1,2, \ldots, n$, and for $z \neq s, W\left(w_{1}(z, s), w_{2}(z, s), \ldots, w_{k}(z, s)\right) \neq 0$ in $D, k=1,2, \ldots, n$. Now it follows from Lemma 6 that equation (1) has property 2. That completes the proof of Theorem 1. 
EXAMPLE 2. Consider the particular case of (5) where $f_{k}(z, s) \equiv 1, k=$ $1,2, \ldots, n$. Then we get the equation

$$
\frac{1}{(z-s)^{n-1}} \frac{d}{d z}(z-s)^{2} \frac{d}{d z} \ldots \frac{d}{d z}(z-s)^{2} \frac{d}{d z} \frac{y(z)}{(z-s)^{n-1}}=0 .
$$

The solutions of this equation are (see (12) and (14)): $1,(z-s)^{2}, \ldots,(z-s)^{n-1}$. Therefore this equation is a factorization of $y^{(n)}(z)=0$.

The equivalent factorization of type (4) is:

$$
y^{(n)}(z)=\left(\frac{d}{d z}+\frac{(n-1)}{z-s}\right) \ldots\left(\frac{d}{d z}-\frac{(n-2 k+1)}{z-s}\right) \ldots\left(\frac{d}{d z}-\frac{(n-1)}{z-s}\right) y(z) .
$$

It follows from Theorem 1 that the equation $y^{(n)}(z)=0$ is disconjugate at two points. Indeed this equation is disconjugate in the whole plane.

REMARK. The adjoint equation of (1) is defined to be

$$
\begin{aligned}
L^{T} v(z)= & (-1)^{n} v^{(n)}(z)+(-1)^{n-1}\left(p_{n-1}(z) v(z)\right)^{(n-1)} \\
& +\cdots+(-1)\left(p_{1} v\right)^{\prime}+p_{0} v=0 .
\end{aligned}
$$

Equation (1) has a solution with the property $(k, n-k)$ if and only if equation (16) has a solution with the property $(k, n-k)$. (The proof is the same as in the real case; see [1, pp. 102-104].) Hence, (1) is disconjugate at two points in $D$ if and only if (16) has the same property. In fact, for every $s \in D$, (1) can be factorized as

$$
\begin{aligned}
\frac{f_{1}(z, s) f_{2}(z, s) \ldots f_{n}(z, s)}{(z-s)^{n-1}} \frac{d}{d z} \frac{(z-s)^{2}}{f_{n}(z, s)} \frac{d}{d z} & \\
& \ldots \frac{d}{d z} \frac{(z-s)^{2}}{f_{2}(z, s)} \frac{d}{d z} \frac{y(z)}{(z-s)^{n-1} f_{1}(z, s)}=0
\end{aligned}
$$

if and only if (16) can be factorized as

$$
\begin{aligned}
L^{T} v(z)=\frac{(-1)^{n}}{(z-s)^{n-1} f_{1}(z, s)} \frac{d}{d z} \frac{(z-s)^{2}}{f_{2}(z, s)} \frac{d}{d z} \\
\ldots \frac{d}{d z} \frac{(z-s)^{2}}{f_{n}(z, s)} \frac{d}{d z} \frac{f_{1}(z, s) \ldots f_{n}(z, s) v(z)}{(z-s)^{n-1}}=0 .
\end{aligned}
$$

3. Construction of equations disconjugate at two points with respect to $\alpha$. We call equation (1) disconjugate at two points with respect to $\alpha$ if and only if no solution which has a zero of multiplicity $k$ at $\alpha$ has an additional zero in $D$ of multiplicity greater than or equal to $n-k, k=1,2, \ldots, n-1$. (Note that (1) is disconjugate at two points in $D$ if and only if it is disconjugate at two points with respect to $\alpha$ for any $\alpha \in D$.)

Using Theorem 1 we obtain a procedure which enables us to build all the equations which are disconjugate at two points with respect to $\alpha$ in $D$ for a given $\alpha$ in $D$. For simplicity we will take $\alpha=0$; this choice does not limit the generality of the discussion. 
LEMMA 7. Consider the equation

$$
\begin{array}{r}
\frac{f_{1}(z) f_{2}(z) \ldots f_{n}(z)}{z^{n-1}} \frac{d}{d z}\left(\frac{z^{2}}{f_{n}(z)} \frac{d}{d z}\left(\ldots \frac{d}{d z}\left(\frac{z^{2}}{f_{2}(z)} \frac{d}{d z}\left(\frac{y(z)}{z^{n-1} f_{1}(z)}\right)\right) \ldots\right)\right) \\
=\left(\frac{d}{d z}+r_{n}(z)\right) \ldots\left(\frac{d}{d z}+r_{k}(z)\right) \ldots\left(\frac{d}{d z}+r_{1}(z)\right) y(z)=0
\end{array}
$$

where $r_{k}(z), k=1,2, \ldots, n$, are regular in $D$ except at $z=0$ and each of them has there a simple pole, $r_{k}(z)=-(n-2 k+1) / z+\ldots$; or, equivalently, let $f_{k}(z)$ be analytic functions, $f_{k}(z) \neq 0$ in $D$ and $f_{k}(0)=1, k=1,2, \ldots, n$.

If (17) can be expanded as

$$
y^{(n)}(z)+q_{n-1}(z) y^{(n-1)}(z)+\cdots+q_{0}(z) y(z)=0,
$$

where the coefficients $q_{i}(z), i=0, \ldots, n-1$, are regular functions in $D$, then the equation is disconjugate at two points in $D$ with respect to the origin.

From now on whenever we mention the coefficients of (17), we mean the coefficients $q_{i}(z)$, after expanding (17) to a linear homogeneous equation (17').

Proof. This follows from the assertion of Theorem 1 for fixed $s, s=0$.

REMARK. The coefficient of $y^{(n-1)}(z)$ in (17) is zero if and only if $r_{1}(z)+r_{2}(z)$ $+\cdots+r_{n}(z) \equiv 0$ or, equivalently, if (17) has the form

$$
\begin{aligned}
\frac{f_{2}^{1 / n} f_{3}^{2 / n} \ldots f_{n}^{(n-1) / n}}{z^{n-1}} \frac{d}{d z} & \frac{z^{2}}{f_{n}(z)} \frac{d}{d z} \\
& \ldots \frac{d}{d z} \frac{z^{2}}{f_{2}(z)} \frac{d}{d z} \frac{f_{2}^{(n-1) / n} f_{3}^{(n-2) / n} \ldots f_{n}^{1 / n}}{z^{n-1}} y(z)=0 .
\end{aligned}
$$

The condition $r_{1}+r_{2}+\cdots+r_{n}=0$ follows immediately from

$$
\begin{aligned}
& \left(d / d z+r_{n}(z)\right) \ldots\left(d / d z+r_{1}(z)\right) y(z) \\
& \quad=y^{(n)}(z)+\left(r_{1}(z)+r_{2}(z)+\cdots+r_{n}(z)\right) y^{(n-1)}(z)+\ldots
\end{aligned}
$$

Let $y_{1}(z), y_{2}(z), \ldots, y_{n}(z)$ be a basis of solutions of $(1)$ and let $W_{n}(z)$ be their Wronskian. The coefficient of $y^{(n-1)}(z)$ in (1) is zero if and only if $W_{n}(z) \equiv$ const. Using the solution (12) (with $s=0$ ), we thus obtain by (13) that

$$
f_{1}^{n}(z) f_{2}^{n-1}(z) \ldots f_{n}(z)=1
$$

EXAMPLE 3. Consider a special case of (18), for $n=2$, where $f_{2}(z) \neq 0$ in $D$, $f_{2}(0)=1$. If $f_{2}^{\prime}(0)=0$, then there exists a meromorphic function $f(z) ; f(z)=1 / z$ $+\ldots, f^{\prime}(z) \neq 0$ in $D$, such that $f^{\prime}(z)=-f_{2}(z) / z^{2}$. (Such $f(z)$ exists only if $f_{2}^{\prime}(0)=0$.) In this case $(18)$ has the form

$$
\sqrt{f^{\prime}(z)} \frac{d}{d z} \frac{1}{f^{\prime}(z)} \frac{d}{d z} \sqrt{f^{\prime}(z)} y(z)=y^{\prime \prime}(z)+\frac{1}{2}\{f, z\} y(z)=0 .
$$

Here $\{f, z\}$ is the Schwarzian derivative of $f(z)$.

$$
\{f, z\}=f^{\prime \prime \prime} / f^{\prime}-\frac{3}{2}\left(f^{\prime \prime} / f^{\prime}\right)^{2}
$$

$\{f, z\}$ is a regular function in $D$, and therefore it follows from Lemma 7 that (19) is disconjugate with respect to the origin. 
In general the coefficients of the linear equation (17) are not regular functions in $D$, but there exist certain relations between the coefficients of the series of $r_{k}(z)$ (or the coefficients of the power series of $\left.f_{k}(z)\right), k=1,2, \ldots, n$, which ensure that (17) has regular coefficients. In this section we develop an algorithm to obtain these relations.

LEMMA 8. The linear equation (17) of order $n$ has regular coefficients if and only if the following solutions are regular functions:

$$
\begin{aligned}
& y_{1}(z)=z^{n-1} f_{1}(z)=v_{1}(z), \\
& y_{2}(z)=z^{n-1} f_{1}(z) \int^{z} \frac{f_{2}(\zeta)}{\zeta^{2}} d \zeta=v_{1}(z) \int^{z} v_{2}(\zeta) d \zeta,
\end{aligned}
$$

(21):

$$
\begin{aligned}
y_{k}(z) & =z^{n-1} f_{1}(z) \int^{z} \frac{f_{2}\left(\zeta_{1}\right)}{\zeta_{1}^{2}} \ldots \int^{\zeta_{k-2}} \frac{f_{k}\left(\zeta_{k-1}\right)}{\zeta_{k-1}^{2}} d \zeta_{k-1} \ldots d \zeta_{1} \\
& =v_{2}(z) \int^{z} v_{2}\left(\zeta_{1}\right) \ldots \int^{\zeta_{k-2}} v_{k}\left(\zeta_{k-1}\right) d \zeta_{k-1} \ldots d \zeta_{1}, \quad k=3, \ldots, n .
\end{aligned}
$$

Proof. If the coefficients of (17) are regular, it is obvious that the solutions (21) are regular functions.

Equation (17) can be written as

$$
\frac{W\left(y_{1}(z), y_{2}(z), \ldots, y_{n}(z), y(z)\right)}{W\left(y_{1}(z), y_{2}(z), \ldots, y_{n}(z)\right)}=0,
$$

since

$$
W\left(y_{1}, y_{2}, \ldots, y_{n}\right)=f_{1}^{n}(z) f_{2}^{n-1}(z) \ldots f_{n}(z) \neq 0 \text { in } D
$$

(see (13) for $s=0$ ). Therefore, if solution (21) is regular, then (17) has regular coefficients.

REMARK. If we want to assure that the solutions $y_{k}(z)$, given by (21), are regular functions, then by the regularity of the given functions $f_{k}(z)$, we have only to verify that no logarithmic terms appear after integration (see (14)).

THEOREM 2. Let $f_{i}(z), i=1,2, \ldots, n$, be analytic functions different from zero in $D:$

$$
f_{i}(z)=1+f_{i, 1} z+\cdots+f_{i, n-1} z^{n-1}+\ldots, \quad i=1,2, \ldots, n .
$$

Then (17) has analytic coefficients in $D$, and it is disconjugate at two points with respect to the origin, if and only if between the $(n-1)^{2}$ first coefficients $f_{i j}, i=$ $2,3, \ldots, n, j=1,2, \ldots, n-1$, there exist $n(n-1) / 2$ relations of the form

$$
\sum_{\nu=0}^{j-1}(-1)^{\nu}\left(\begin{array}{c}
j-1 \\
\nu
\end{array}\right) f_{i-\nu, j}=\phi_{i, j} ; \quad \begin{aligned}
& j=1,2, \ldots, n-1 \\
& i=j+1, \ldots, n
\end{aligned} ; \quad\left(\begin{array}{l}
\nu \\
0
\end{array}\right)=1 .
$$

Here $\phi_{i, j}=0$ for $j=1,2, \ldots, 6$ and, for $j>7, \phi_{i, j}$ is a polynomial in $f_{i^{\prime}, j^{\prime}}$, $i^{\prime}=i-j+1, \ldots, i, j^{\prime}=1, \ldots, j-1$. 
Moreover, all the equations (1) which are disconjugate at two points with respect to the origin can be factorized in the form (17), where the functions $f_{i}(z)$ are analytic and different from zero in $D, i=1,2, \ldots, n$, and their first coefficients fulfill the relations (23).

Proof. Let

$$
\begin{aligned}
& v_{i}(z)=\frac{f_{i}(z)}{z^{2}}=\frac{1}{z^{2}}+\frac{v_{i,-1}}{z}+v_{i, 0}+v_{i, 1} z+v_{i, 2} z^{2}+\ldots ; \\
& v_{i, j-2}=f_{i, j} ; \quad i=2, \ldots, n ; j=1,2, \ldots
\end{aligned}
$$

We write explicitly the first relations of (23) which we want to prove.

$$
\begin{aligned}
v_{i,-1} & =0 ; & i=2,3, \ldots, n, \\
v_{i, 0}-v_{i-1,0} & =0 ; & i=3,4, \ldots, n, \\
v_{i, 1}-2 v_{i-1,1}+v_{i-2,1} & =0 ; & i=4,5, \ldots, n, \\
v_{i, 2}-3 v_{i-1,2}+3 v_{i-2,2}-v_{i-3,2} & =0 ; & i=5, \ldots, n .
\end{aligned}
$$

In order that the solution $y_{i}=v_{1} \int v_{2} \ldots \int v_{i}$ will not contain logarithmic terms, $i=2,3, \ldots, n$, we require each step of the integration $\int v_{i-j} \int v_{i-j+1} \int \ldots \int v_{i}$ to be free of logarithmic terms.

The first step: $\int v_{i} d \zeta$ will not contain a logarithm if $v_{i,-1}=0, i=2, \ldots, n$, and this is the first relation of $\left(23^{\prime}\right)$.

Now the second step is

$$
\int v_{i-1} \int v_{i}=\int\left(-\frac{1}{z^{3}}+\frac{-v_{i-1,0}+v_{i, 0}}{z}+\ldots\right) d z
$$

and it will not contain a logarithmic term if $v_{i, 0}-v_{i-1,0}=0, i=3, \ldots, n$; this is the second relation of $\left(23^{\prime}\right)$.

Denote $v_{i, 0}=v_{0}, i=2, \ldots, n$; we obtain

$$
\int v_{i-2} \int v_{i-1} \int v_{i}=\int\left(\frac{1}{2 z^{4}}+\frac{v_{0}}{2 z^{2}}+\frac{-2 v_{i-1,1}+v_{i, 1}+v_{i-2,1}}{z}+\ldots\right) d z,
$$

which leads to the third relation of $\left(23^{\prime}\right)$.

The next step is

$$
\begin{aligned}
& \int v_{i-3} \int v_{i-2} \int v_{i-1} \int v_{i} \\
& =\int\left(-\frac{1}{6 z^{5}}+\frac{2 v_{0}}{3 z^{2}}+\frac{v_{i-3,1}}{6 z}+\left(\frac{v_{i-2,2}}{2}+\frac{v_{i, 2}}{6}-\frac{v_{i-1,2}}{2}-\frac{v_{i-3,2}}{6}\right) \frac{1}{z}+\ldots\right) d z,
\end{aligned}
$$

which leads to the fourth relation from $\left(23^{\prime}\right)$.

The algorithm to get (23) is obvious now. The calculation shows that relations (23) are necessary and sufficient for the solutions of (17) to have no logarithmic terms. Hence, from Lemmas 7 and 8 we get that they are also necessary and sufficient for (17) to have regular analytic coefficients and to be disconjugate at two points with respect to the origin. 
When (1) is disconjugate at two points with respect to the origin, it follows from Theorem 1 that it has the factorization (17), where the functions $f_{k}(z), k=$ $1,2, \ldots, n$, are analytic and different from zero in $D$. It follows from what we have proved above that the coefficients of the Taylor expansion of the functions $f_{k}(z)$ satisfy (23). That completes the proof.

REMARKS. (i) In Theorem 2 we not only build all the equations whose solutions satisfy certain zero properties in $D$, but we also do it in a form that enables us to solve them (see (21)). (ii) We conjecture $\phi_{i j}=0$ for all $j=1,2, \ldots, n-1 ; i=j+$ $1, \ldots, n$.

EXAmPLe 4. Let $f(z), f(z)=1 / z+\ldots$, be analytic in $D$ except at $z=0$, and $f^{\prime}(z) \neq 0$ in $D$. Then the equation

$$
\left(f^{\prime}(z)\right)^{(n-1) / 2} \frac{d}{d z} \frac{1}{f^{\prime}(z)} \frac{d}{d z} \ldots \frac{d}{d z} \frac{1}{f^{\prime}(z)} \frac{d}{d z}\left(f^{\prime}(z)\right)^{(n-1) / 2} y(z)=0
$$

is disconjugate at two points with respect to the origin.

Proof. A detailed discussion of this Fano equation (25) is given by Lavie [4, pp. $446-450]$. It follows from (21) that the general solution of (25) is

$$
\begin{aligned}
y(z) & =C_{1} u^{n-1}(z)+C_{2} u^{n-2}(z) v(z)+\cdots+C_{n} v^{n-1}(z) \\
& =\prod_{i=1}^{n-1}\left(m_{i} u(z)-n_{i} v(z)\right)
\end{aligned}
$$

where $u(z)=f(z) / \sqrt{f^{\prime}(z)}, v(z)=1 / \sqrt{f^{\prime}(z)}$ is a basis of solutions of (19).

Hence, we get that a solution of (25) has $n$ zeros in $D$ if and only if one of the solutions of (19), $m_{i_{0}} u(z)-n_{i_{0}} v(z)$, has two zeros in $D$. This, and the fact that (19) is disconjugate at two points with respect to the origin (see Example 3), completes the proof.

In Theorem 2 we have to assume that the regular functions $f_{i}(z)$, which satisfy the relations (23), do not vanish in $D$. To avoid this we formulate the analogous result in terms of the functions $r_{k}(z)$.

THEOREM 3. Let $h_{1}(z), h_{2}(z), \ldots, h_{n}(z)$ be analytic functions in $D$. Then the equation

$$
\begin{aligned}
\left(\frac{d}{d z}+\frac{(n-1)}{z}+\sum_{\nu=0}^{n-2} h_{n \nu} z^{\nu}+z^{n-1} h_{n}(z)\right) \\
\ldots\left(\frac{d}{d z}-\frac{(n-2 k+1)}{z}+\sum_{\nu=0}^{n-2} h_{k \nu} z^{\nu}+z^{n-1} h_{k}(z)\right) \\
\ldots\left(\frac{d}{d z}-\frac{(n-1)}{z}+\sum_{\nu=0}^{n-2} h_{1 \nu} z^{\nu}+z^{n-1} h_{1}(z)\right) y(z) \equiv 0
\end{aligned}
$$


has analytic coefficients in $D$, and is disconjugate at two points with respect to the origin, if and only if between the $n(n-1)$ first coefficients $h_{i j}, i=1,2, \ldots, n$, $j=0, \ldots, n-2$, exist $n(n-1) / 2$ relations of the form

$$
\begin{aligned}
& \sum_{\nu=0}^{j}(-1)^{\nu}\left(\begin{array}{l}
j \\
\nu
\end{array}\right) H_{i-\nu, j}=\theta_{i, j}, \quad j=0, \ldots, n-2 ; \\
& i=j+2, \ldots, n ; \quad\left(\begin{array}{l}
\nu \\
0
\end{array}\right)=1 .
\end{aligned}
$$

Here $H_{i, j}=-h_{i, j}+h_{i-1, j}$ and $\theta_{i j}=0$ for $j=0,1, \ldots, 5$ and, for $j \geqslant 6, \theta_{i j}$ is $a$ polynomial in $H_{i^{\prime} j^{\prime}} ; i^{\prime}=i-j, \ldots, n, j^{\prime}=1, \ldots, j-1$.

Moreover all the equations (1) which are disconjugate at two points with respect to the origin can be factorized in the form (26), where the functions $h_{i}(z)$ are analytic in $D, i=1,2, \ldots, n$, and the coefficients $h_{i j}$ fulfill relations (27).

Proof. This theorem is analogous to Theorem 2. We have (see Lemma 4)

$$
\begin{aligned}
& \frac{f_{k}(z)}{z^{2}}= \exp \int^{z}\left(r_{k-1}(\zeta)-r_{k}(\zeta)\right) d \zeta \\
&=\exp \int^{z}\left(-\frac{2}{\zeta}+\left(h_{k-1,0}-h_{k, 0}\right)+\left(h_{k-1,1}-h_{k, 1}\right) \zeta+\ldots\right) d \zeta, \\
& k=2, \ldots, n .
\end{aligned}
$$

Denote $H_{k, j}=h_{k-1, j}-h_{k j}$; then

$$
\frac{f_{k}(z)}{z^{2}}=\frac{1}{z^{2}} \sum_{\nu=0}^{\infty} f_{k, \nu} z^{\nu}=\frac{1}{z^{2}} \exp \sum_{\nu=0}^{\infty} \frac{H_{k, \nu}}{\nu+1} z^{\nu+1} ; \quad f_{k 0}=1 .
$$

Hence

$$
\sum_{\nu=0}^{\infty} \frac{H_{k, \nu} z^{\nu+1}}{\nu+1}=\log \left(\sum_{\nu=0}^{\infty} f_{k, \nu} z^{\nu}\right)
$$

and by differentiation we get

$$
\left(\sum_{\nu=0}^{\infty} H_{k, \nu} z^{\nu}\right)\left(\sum_{\nu=0}^{\infty} f_{k, \nu} z^{\nu}\right)=\sum_{\nu=1}^{\infty} \nu f_{k, \nu} z^{\nu-1}=\sum_{\nu=0}^{\infty}(\nu+1) f_{k, \nu+1} z^{\nu}
$$

Therefore

$$
(p+1) f_{k, p+1}=\sum_{\nu=0}^{p} H_{k, 0} f_{k, p-\nu} .
$$

From (28) and (23) we obtain relations (27). That completes the proof of Theorem 3.

Corollary. Equations (27) of Theorem 3 show that the coefficients $h_{i 0}, i=$ $1,2, \ldots, n$, in (26) are determined by one arbitrary parameter. The coefficients $h_{i 1}$, $i=1,2, \ldots, n$, are determined by two parameters, and so on. Hence, the coefficients $h_{i, k-1}, i=1,2, \ldots, n, k=1,2, \ldots, n-1$, are determined by $n(n-1) / 2$ parameters. 
Therefore, all the equations that are disconjugate at two points with respect to $z=0$ depend on $n$ arbitrary analytic functions $h_{1}(z), h_{2}(z), \ldots, h_{n}(z)$ written in (26) and on $n(n-1) / 2$ arbitrary parameters that determine the coefficients $h_{i, k-1}$.

4. Second and third order equations. We use the results of the previous sections for some special cases. First we consider second order equations.

As a special case of Theorem 3 (see also the remark after Lemma 7), we obtain that the equation $y^{\prime \prime}(z)+p(z) y(z)=0$ is disconjugate with respect to $\alpha, \alpha \in D$, if and only if it can be written in the form

$$
\left(\frac{d}{d z}-\sigma(z)\right)\left(\frac{d}{d z}+\sigma(z)\right) y(z)=y^{\prime \prime}(z)+\left(\sigma^{\prime}(z)-\sigma^{2}(z)\right) y(z)=0,
$$

where $\sigma(z)=-1 /(z-\alpha)+(z-\alpha) h(z, \alpha)$ and $h(z, \alpha)$ is an analytic function. Thus we obtain the known result that the equation $y^{\prime \prime}(z)+p(z) y(z)=0$ is disconjugate in $D$ if and only if every solution of the Riccati equation $\sigma^{\prime}(z)-\sigma^{2}(z)=$ $p(z)$ has only one singularity in $D$.

EXAMPLE 5 . When $p(z) \equiv 1$, the general solution of the equation $\sigma^{\prime}(z)-\sigma^{2}(z)=$ 1 (i.e. $\left.d z / d \sigma=1 /\left(1+\sigma^{2}\right)\right)$ is $z=\operatorname{arctg} \sigma+c$. The solution whose Laurent expansion begins with $-1 /(z-\alpha)$ is $\sigma(z)=-\operatorname{ctg}(z-\alpha)$. Thus we obtain

$$
y^{\prime \prime}(z)+y(z)=\left(\frac{d}{d z}+\operatorname{ctg}(z-\alpha)\right)\left(\frac{d}{d z}-\operatorname{ctg}(z-\alpha)\right) y(z)=0 .
$$

This equation is disconjugate with respect to $\alpha$, in a domain where $\operatorname{ctg}(z-\alpha)$ is regular, $z \neq \alpha$, for example in the vertical strip $\operatorname{Re} \alpha-\pi<\operatorname{Re} z<\operatorname{Re} \alpha+\pi$. Hence the equation is disconjugate in any vertical strip of width $\pi$.

As a special case, for $n=2$, of Theorem 2 (see also the remark and example after Lemma 7), we obtain that the equation $y^{\prime \prime}(z)+p(z) y(z)=0$ is disconjugate with respect to the origin if and only if it can be written in the form:

$$
\sqrt{f^{\prime}(z)} \frac{d}{d z} \frac{1}{f^{\prime}(z)} \frac{d}{d z} \sqrt{f^{\prime}(z)} y(z)=y^{\prime \prime}(z)+\frac{1}{2}\{f, z\} y(z)=0
$$

where the analytic function $f(z)=1 / z+\ldots$ is regular, except at $z=0$, and $f^{\prime}(z) \neq 0$ in $D$. This result yields the following proposition.

Proposition 1. The equation $y^{\prime \prime}(z)+p(z) y(z)=0$ is disconjugate in $D$ if and only if it can be written in the form (19) where $f(z)$ is univalent in $D, f(z)=1 / z+\ldots$

Proof. Two independent solutions of (19) are $y_{1}(z)=1 / \sqrt{f^{\prime}(z)}$, $y_{2}(z)=f(z) / \sqrt{f^{\prime}(z)}$. Thus, the equation is disconjugate if and only if for any $z_{1}$, $z_{2} \in D, z_{1} \neq z_{2}$, the determinant

$$
\left|\begin{array}{ll}
y_{1}\left(z_{1}\right) & y_{2}\left(z_{1}\right) \\
y_{1}\left(z_{2}\right) & y_{2}\left(z_{2}\right)
\end{array}\right|=\frac{f\left(z_{2}\right)-f\left(z_{1}\right)}{\sqrt{f^{\prime}\left(z_{1}\right) f^{\prime}\left(z_{2}\right)}}
$$

is different from zero, i.e., $f\left(z_{1}\right) \neq f\left(z_{2}\right)$. Hence, the equation is disconjugate if and only if $f(z)$ is univalent. 
Proposition 1 is a well-known result of Nehari [5], which states that the equation $y^{\prime \prime}(z)+p(z) y(z)=0$ is disconjugate in $D$ if and only if $f(z)=y_{2}(z) / y_{1}(z)$ is a univalent function in $D$, where $y_{1}(z)$ and $y_{2}(z)$ are two arbitrary independent solutions.

We now consider third order equations.

LEMMA 9. The equation $y^{\prime \prime \prime}(z)+p_{1}(z) y^{\prime}(z)+p_{0}(z) y(z)=0$ is disconjugate at two points with respect to the origin if and only if it can be written in the form:

$$
\begin{aligned}
L y(z) & =\left(f^{\prime}(z)\right)^{1 / 3}\left(g^{\prime}(z)\right)^{2 / 3} \frac{d}{d z} \frac{1}{g^{\prime}(z)} \frac{d}{d z} \frac{1}{f^{\prime}(z)} \frac{d}{d z}\left(f^{\prime}(z)\right)^{2 / 3}\left(g^{\prime}(z)\right)^{1 / 3} y(z) \\
& =y^{\prime \prime \prime}(z)+\left[\{f, z\}+\{g, z\}+\left(f^{\prime \prime} / f^{\prime}-g^{\prime \prime} / g^{\prime}\right)^{2} / 6\right] y^{\prime}(z)+R(z) y(z) \\
& =0, \\
R(z) & =\left(f^{\prime}(z)\right)^{1 / 3}\left(g^{\prime}(z)\right)^{2 / 3} \frac{d}{d z} \frac{1}{g^{\prime}(z)} \frac{d}{d z} \frac{1}{f^{\prime}(z)} \frac{d}{d z}\left(f^{\prime}(z)\right)^{2 / 3}\left(g^{\prime}(z)\right)^{1 / 3},
\end{aligned}
$$

where $f(z)=1 / z+a z+\ldots, g(z)=1 / z+a z+\ldots$, are analytic functions in $D$ for $z \neq 0$, and $g^{\prime}(z) \neq 0, f^{\prime}(z) \neq 0$ in $D$.

Proof. This lemma is a special case of Theorem 2, for $n=3$; we use the remark after Lemma 7, and we denote $g^{\prime}(z)=-f_{3}(z) / z^{2}, f^{\prime}(z)=-f_{2}(z) / z^{2}$.

For example, if $g(z)=f(z)$ then (30) is the Fano equation which we have considered in Example 4 (see [4, p. 441]):

$$
\begin{aligned}
L y(z) & =f^{\prime}(z) \frac{d}{d z} \frac{1}{f^{\prime}(z)} \frac{d}{d z} \frac{1}{f^{\prime}(z)} \frac{d}{d z} f^{\prime}(z) y(z) \\
& =y^{\prime \prime \prime}(z)+2\{f, z\} y^{\prime}(z)+\{f, z\}^{\prime} y(z)=0 .
\end{aligned}
$$

Using Lemma 9 we obtain our two final results.

Proposition 2. The equation $y^{\prime \prime \prime}(z)+p_{1}(z) y^{\prime}(z)+p_{0}(z) y(z)=0$ is disconjugate at two points in $D$ if and only if the following conditions are satisfied:

(1) the condition of Lemma 9;

(2) for any $z_{0} \in D, z_{0} \neq 0$, and for $z \neq z_{0}, W\left(\left(k(z)-k\left(z_{0}\right)\right),\left(f(z)-f\left(z_{0}\right)\right)\right) \neq 0$ in $D$, where $k(z)=2 \int^{z} f^{\prime}(\zeta) g(\zeta) d \zeta$.

Proof. The first condition follows by Lemma 9. For the second condition we write the solutions of (30):

$$
\begin{aligned}
& y_{1}(z)=1 /\left(\left(f^{\prime}(z)\right)^{2 / 3}\left(g^{\prime}(z)\right)^{1 / 3}\right) \stackrel{\text { def }}{\equiv} T(z), \\
& y_{2}(z)=f(z) T(z) \\
& y_{3}(z)=2 T(z) \int^{z} f^{\prime}(\zeta) g(\zeta) d \zeta=T(z) k(z) .
\end{aligned}
$$

Note that $T(z)$ has a double zero at $z=0$ and is different from zero otherwise. 
Equation (30) is disconjugate at two points if and only if for any $z_{0} \in D, z_{0} \neq 0$, and for any $z \neq z_{0}$ we have:

$$
\left|\begin{array}{lll}
y_{1}\left(z_{0}\right) & y_{2}\left(z_{0}\right) & y_{3}\left(z_{0}\right) \\
y_{1}(z) & y_{2}(z) & y_{3}(z) \\
y_{1}^{\prime}(z) & y_{2}^{\prime}(z) & y_{3}^{\prime}(z)
\end{array}\right|=T\left(z_{0}\right) T^{2}(z)\left|\begin{array}{cc}
f(z)-f\left(z_{0}\right) & k(z)-k\left(z_{0}\right) \\
f^{\prime}(z) & k^{\prime}(z)
\end{array}\right| \neq 0 .
$$

This completes the proof.

Proposition 3. The equation $y^{\prime \prime \prime}(z)+p_{1}(z) y^{\prime}(z)+p_{0}(z) y(z)=0$ is disconjugate in $D$ if and only if the following conditions are satisfied:

(1) the condition of Lemma 9;

(2) $f(z)$ is a univalent function in $D$;

(3) for any $z_{0} \in D, z_{0} \neq 0$, the function $\left(k(z)-k\left(z_{0}\right)\right) /\left(f(z)-f\left(z_{0}\right)\right)$ is univalent in $D, k(z)=2 \int^{z} f^{\prime}(\zeta) g(\zeta) d \zeta$.

Proof. The first condition follows by Lemma 9.

Now (30) is disconjugate if and only if the following conditions are satisfied:

(1) For any $z_{1}, z_{2} \in D, z_{1} \neq z_{2}$, we have

$$
\begin{aligned}
\left|\begin{array}{lll}
y_{1}(0) & y_{2}(0) & y_{3}(0) \\
y_{1}\left(z_{1}\right) & y_{2}\left(z_{1}\right) & y_{3}\left(z_{1}\right) \\
y_{1}\left(z_{2}\right) & y_{2}\left(z_{2}\right) & y_{3}\left(z_{2}\right)
\end{array}\right| & =\left|\begin{array}{ccc}
0 & 0 & -1 \\
T\left(z_{1}\right) & T\left(z_{1}\right) f\left(z_{1}\right) & T\left(z_{1}\right) k\left(z_{1}\right) \\
T\left(z_{2}\right) & T\left(z_{2}\right) f\left(z_{2}\right) & T\left(z_{2}\right) k\left(z_{2}\right)
\end{array}\right| \\
& =-T\left(z_{1}\right) T\left(z_{2}\right)\left(f\left(z_{2}\right)-f\left(z_{1}\right)\right) \neq 0
\end{aligned}
$$

$\left(y_{1}, y_{2}, y_{3}\right.$ are defined in (31)).

The first equality is obtained since $k(z)=1 / z^{2}+k_{0}+\ldots$, and $y_{3}(0)=-1$. That gives the second condition of the proposition.

(2) For any $z_{0}, z_{1}, z_{2} \in D$, we have

$$
\left|\begin{array}{lll}
y_{1}\left(z_{0}\right) & y_{2}\left(z_{0}\right) & y_{3}\left(z_{0}\right) \\
y_{1}\left(z_{1}\right) & y_{2}\left(z_{1}\right) & y_{3}\left(z_{1}\right) \\
y_{1}\left(z_{2}\right) & y_{2}\left(z_{2}\right) & y_{3}\left(z_{2}\right)
\end{array}\right|=T\left(z_{0}\right) T\left(z_{1}\right) T\left(z_{2}\right)\left|\begin{array}{ll}
f\left(z_{1}\right)-f\left(z_{0}\right) & k\left(z_{1}\right)-k\left(z_{0}\right) \\
f\left(z_{2}\right)-f\left(z_{0}\right) & k\left(z_{2}\right)-k\left(z_{0}\right)
\end{array}\right| \neq 0 .
$$

Since $T(z) \neq 0$ for $z \neq 0$, and $f(z)$ is univalent, this is equivalent to the third condition in the proposition.

Remarks. (1) Proposition 3 is a special case, for $n=3$, of the following theorem of M. Lavie [3]:

Equation (1) is disconjugate in $D$ if and only if the function $f\left(z, a_{1}, a_{2}, \ldots, a_{n-2}\right)$ $=y_{1}(z) / y_{2}(z)$ is univalent in $D$ for any choice of two linearly independent solutions $y_{1}(z)$ and $y_{2}(z)$ which vanish on any given set $\left(a_{1}, a_{2}, \ldots, a_{n-2}\right)$ of $n-2$ points of $D$.

(2) If the equation $y^{\prime \prime \prime}+p_{1} y^{\prime}+p_{0} y=0$ is disconjugate then the function $k(z)$ of Proposition 3 is a 2-valent function in $D$. That follows from the fact that in Proposition 3 we can replace condition (3) by the requirement that for any 
arbitrary constants $C_{1}$ and $C_{2}$ the function $C_{1} f(z)+C_{2} k(z)$ is 2-valent in $D$. This remark is related to a result of $\mathrm{Kim}[2]$ :

Equation (1) is disconjugate in $D$ if and only if any combination of $y_{3} / y_{1}, y_{2} / y_{1}$ is a 2 -valent function in $D$, where $y_{1}, y_{2}, y_{3}$ are three independent solutions of (1). Kim proves this result for equations of arbitrary order $n$.

\section{REFERENCES}

1. W. A. Coppel, Disconjugacy, Lecture Notes in Math., vol. 220, Springer-Verlag, Berlin and New York, 1971.

2. W. J. Kim, The Schwarzian derivative and multivalence, Pacific J. Math. 31 (1969), 717-724.

3. M. Lavie, The Schwarzian derivative and disconjugacy of $n$-th order linear differential equations, Canad. J. Math. 21 (1969), 235-249.

4. _ Disconjugacy of linear differential equations in the complex domain, Pacific J. Math. 32 (1970), 435-457.

5. Z. Nehari, The Schwarzian derivative and schlicht functions, Bull. Amer. Math. Soc. 55 (1949), 545-551.

6. A. C. Peterson, On the sign of Green's function beyond the interval of disconjugacy, Rocky Mountain J. Math. 3 (1973), 41-51.

7. G. Polya, On the mean value theorem corresponding to a given linear homogeneous differential equation, Trans. Amer. Math. Soc. 24 (1924), 312-324.

Department of Mathematics, Technion-I.I.T., Haifa, IsRael 